\title{
AMÉRICA LATINA Y LA PRÁCTICA DE LA LITERATURA COMPARADA ${ }^{3}$
}

Dr. Biagio D'/ngclo

\section{Algunas definiciones}

Es necesario en este caso contestar a una de las preguntas más dificiles para los estudiosos que corresponde no sólo a "¿qué es la literatura comparada?», sino también "comparar, ¿qué?», "¿̨qué se compara?», "ęcuántas literaturas, una, dos o tres?». Son preguntas "necesarias" y que exigen una tentativa de respuesta. Una de las maneras más virtuosas es ofrecer una definición de la literatura comparada que, ya de por sí, representaría un capitulo a parte, muy complejo, de la histotia del término. Intentemos un resumen: este término que, como todas las etiquetas convencionales, se revela poco clatificador fue promovido por primera vez en el mundo académico en 1832, durante un curso de «Histoire comparative des litteratures» ('Historia comparada de las literaturas') dictado por Jean-Jacques Ampère en la Sorbona de París. El participio comparút, que será utilizado más tarde como la versión más acreditada, será copiado o retomado por todas las lenguas románicas, mientras en las lenguas anglosajonas se hablará de comparative o de vergleichend, forma adjetival que se utilizará también en ruso, en húngaro y en holandés. Sin embargo, los alemanes, que tendrían con el fundamental aporte de Goethe un rol decisivo por la difusión de la literatura comparada, con la idea utópica de una welliteratur ('una literatura del mundo'), adoptarán el término más

\footnotetext{
'Este articulo es la trascripción de una ponencia ofrecida por el Autor en el "Centro de Esnudios Literarios Antonio Comejo Polar" de Lama, el 30 de setiembre de 2002.
} 


\section{Biagio D'Angelo}

feliz de vergleichende Literatuknissenschaft, es decir, la 'ciencia literatia comparada', en que se resalta no tanto la literatura (en cuanto textos, crítica, la umaterialidads de lo literario) sino la (metodología científicas que se utiliza para el estudio de las literaturas desde un punto de vista internacional. Entonces, la literatura comparada es, antes de todo, un método que, de acuerdo con Claudio Guillén, podriamos definir como kuna cierta tendencia o rama de la investigación literaria que se ocupa del estudio sistemático de conjuntos supranacionales», Guillén habla de supranacionalidad y no de internacionalidad, porque declara que la identidad de la literatura comparada eno depende solamente de la actitud o postura del observador. Es fundamental la contribución palpable a la historia, o al concepto de literatura, de unas clases y categotías que no son meramente nacionalesw ${ }^{2}$. Seria suficiente pensar en ciertos géneros específicos y universales como la tragedia o ciertos procédimientos poéticos como la elegia, la rima, la oda $o$, finalmente, ciertas corrientes culturales que se han vuelto movimientos literarios universales como, por ejemplo, el Romanticismo. Asi Guillén subraya que wel punto de arranque no lo constituyen las literaturas nacionales, nit las interrelaciones que hubo entre ellasw. Se podria afirmar en definitiva que se trata de una visión cósmica, una percepción de la supranacionalidad como factor participante del Geist ('espiritu') idealista alemán ${ }^{3}$. En el fondo, la literatura comparada es una tensión, un deseo, una actividad frente a otras actividades, una tensión que es su vocación principal, la tentativa de realización del sueño utópico goethiano de estudiarla diteratum del mundon (welliteratur), una vocación y una tensión respecto de la unidad que son ahora más que nunca importantísimos factores de la vida cultural y, quizás por esta misma razón, elementos incómodos en un mundo globalizado. Según una expresión de Adrián Marino:

\footnotetext{
${ }^{2}$ C. Guillén, Entre lo ana y lo diversa. Introduction a la Litenatura rompanada, Barcelona, Editorial Crítica, $1985, \mathrm{p}, 13$.

"De aqui en adclante retomo algunas reflexiones que ya han sido publicadas en "La literatura comparadar un desafio y una necesidad" en Homemoje a Luis jaime Cismeror, Eduardo Hoplkins, ed., vol. 11, Lama: Fondo Editurial de la PUCT, 2002, pp. 813-825.

"Véase H. Birus, "Main Features of Goethe's Conception of Wodd Literature" en Companative

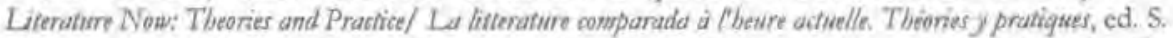
Tờōsy de Zepernek, Milan Y. Dimic and Trene Sywenky. Pans. Honoré Charnpion, 1999, pp. 31-40.
} 


\section{AMÉRICA LATINA Y LA PRÃCTICA DE LA LITERATURA COMPARADA}

Esta disciplina [la literatura comparada] está llamada a adoptar una posición crítica y combativa, a implicarse directamente en las grandes controversias ideológicas de nuestra época ${ }^{5}$.

Más que nunca actual, la militancia sugerida por Marino sirve justamente para desenterrar una metodologia en el ámbito de los estudios literarios, tan puestos en entredicho por la globalización y la comercialización del arte. Los problemas que estamos tratando podrían parecer además insuperables, pero la conciencia de la importancia de un discurso crítico sobre la literatura comparada nos permite percibir o bien la necesidad del estudio comparado de la literatura, o el quid último, la plusvalía que el arte ofrece al espiritu humano. Como tecuerda Octavio Paz: 〈da obra de arte nos deja entrever, por un instante, el allá en el aqui, el siempre en el ahoras. Para revivir y reafrontar la literatura como una especie de. alba de un nuevo humanismo, en in periodo de abstracción filosófica y superficialidad psicológica, tecnicismos lingüísticos y vaguedades sociológicas, sobre todo en el ensayo literario, ocurre una especial disposición de ánimo:

lo que le permite [al comparatista] acometer semejante empresa es la conciencia de unas tensiones entre lo local y lo universal; o si se prefiere, entre lo particular y lo general. Digo local - lugar-y no nación - nacionalidad, pais, región, ciudad-porque conviene destacar aquellos conceptos extremos que encierran una setic de oposiciones generales, aplicables a situaciones diferentes: entre la circiunstancia y el mundo (los mundos); entre lo presente y lo ausente; la experiencia y su sentido; el yo y cuanto le es ajeno; lo percibido y lo anhelado; lo que hay y lo que deberia haber; lo que esta y lo que es?.

Sin esta condición poética y existencial, el compatatista no podrả nunca recoger aquella tensiọ́n (resuelta y no) que se encuentra más allá đẹl fenómeno

\footnotetext{
'A Marine, Etiemble ate le comparatisme militam, Paris, Gallimard, 1982, p. 7: C.ette discipline fla littérature comparéel est appelée à adopter ane postion critique y combative, d simpliques. directement dans les grandes controverses idéologiques de notre époque

"O. Paz, Pintado en Mexcipo, El Pais, Madrid, 7 noviembre 1983, p. 21.

${ }^{7}$ C. Guilér, up cit, p. 15
} 


\section{Biagía D'Angelo}

literario y que está detrás del bastidor de la poética. De todas estas sugerencias se ve además cómo la literatura comparada privilegia todas las literaturas, porque todas representan ciertamente la tierra, el pueblo y el momento de la producción artística, pero también es verdad que todas son superadas, en el instante mismo de la realización, por un elemento misterioso que se escapa a cualquier etiqueta y que sublima el gesto del artista o del escritot. Este elemento misterioso no es objeto de la literatura comparada, porque éste es el estudio de los sistemas literarios supranacionales, que acompaña como faro el recorrido del crítico y el descubrimiento de lo que es común al hecho literario y lo que explica la diversidad.

La idea de una literatura que encuentra (o parte de) una unidad para descifrar (o reunir) una diversidad es en cierto modo una idea romántica. Alexandre Cioranescu, que se limitaba a hablar de una urepública europea de las letrasn, reconocía que podía vislumbrar:

en ciertos espiritus románticos, caracterizados por la excepcional amplirud de su visión histórica, la idea de una unidad de fondo de todas las literaturas, por encima de las fronteras de los pueblos y de sus idiomas ${ }^{b}$.

Hoy la restricción al mundo europeo de una república de las letras se pone profundamente en entredicho; justamente, el hecho de referirse a Europa seria como analizar un microcosmos analizado y domesticado. El eurocentrismo hacia el que se lanzan la mayoria de los estudiosos actuales tiene que ser revisado y redescubierto a través del hallazgo de las nuevas literaturas, donde enuevasn no Lleva en sí exclusivamente la connotación de diteraturas recién nacidasn (es decir, la materia de los análisis upostcolonialesw), sino de litetaturas de larga historia y de complejo desarrollo, sofocadas o jerarquizadas (puestas en segundo plano) por las literaturas europeas. Asi, la literatura comparada puede ser interpretada como la tentativa de confrontar, reunificar, analizar las creaciones artísticas (no necesariamente sólo literarias) que tienen en comun el estudio del Ser y de la Alteridad, en todos los liggares y momentos históricos, hasta los más dispares y

'A. Cioranescu, Prinajoios de líenutura companuda, La Universidad de laguna 1964, p. 18. 


\section{AMÉRICA LATINA Y LA PRĂCTICA DE LA LITERATURA COMPARADA}

lejanos. No sería excraño, pero sí sorprendente, recoger aquella traza común inconfundible que caracteriza la necesidad del hombre de expresarse a través de las formas artísticas.

Por parte de los países de las unuevas» literaturas, en el sentido más amplio del término, la literatura comparada exige un desafio: inaugurado un proceso de integración y de apertura al mundo, la literatura comparada, como metodología de conocimiento de un aspecto de la realidad, se convierte, por eso, en una de las vías privilegiadas para imponer no sólo una conciencia nacional, de pueblo, a nivel internacional, sino también para reconocer y favorecer la importancia de la práctica de la líteratura como factor de conocimiento y de pertenencia frente al tiempo y a la historia.

Y además, todo el conjunto de estudios, temáticas y perspectivas que se condensan bajo el título de sliteranura comparadas es esencial para la comprensión de las culturas de aquellos paises en los cuales el vacio del comparativismo ha creado un aislamiento consiguiente y explicable. La literatura comparada podria operar un auténtico du ut des entre la imagen cultural nacional y la imagen del mundo, entre la idea de litematura cerrada que nace de un concepto de nación puramente sectario y la idea de una pertenencia a una litecatura universal que presenta trazas singulares que son comunes a todos los pueblos.

Asi se vuelve indispensable repensar en el papel de América Latina en los estudios culturales $y$ literarios relacionados con la literatura comparada. En este último ámbito, la cultura latinoamericana se ha quedado casi permanentemente en los márgenes. El cambio de historia de la literatura a estudios culturales, que ha marcado las universidades norteamericanas, ha significado para Latinoamérica no tanto una exclusión, sino una percepción más conciente y profunda del fenómeno literario y su función profética en la ufórmula latinoamericana" de alcanzar al mundo, de expresar una voz que habia sido tácitamente suprimida y que los literatos latinoumericanos habían aceptado sin fuertes polémicas. Mario Valdés se pregunta el porqué de tal persistencia conservadora đe parte de la crítica comparatista en considerar Latinoamérica como «imposibles a la práctica comparatista. Aunque una de las razones de esta dificultad podria ser localizada en la persistencia de un discurso hegemónico oecidental, no se puede esconder la exigencia de que una formulación estándar de la literatura comparada requeria textos originalmente compuestos, por lo menos, en dos idiomas distintos. 


\section{Biagía D'Angelo}

Además, se añade otra idea errada y conservadora de una Hispanoamérica que cubre naciones del hemisferio sur y excluye todo el átea caribeña. Entonces, la comparación literaria asume un aspecto polémico y original en la consideración de las múltiples culturas y literaturas que ocupan el espacio lingüistico hispanoportugués. No considero en este trabajo las culturas asíllamadas minoritarias (cultura quechua, aimara, maya, azteca, etc...) por ser un problema grave y al cual sería necesario dedicar una atención mucho más detallada, Como sugiere Maria Elena de Valdés:

Latin America is a fictional space, forever in between the hegemonic domination of North America and nostalgia for Europe. This inbetween space is populated with voices of such diversity that the wildest fabulations of the first European visitors are but a remote and pale simulacrum, Latin America does not only speak in Spanish and Portuguese, but also in Creole and in Quechua, Nahuatl, Guarani [... $]^{5}$

Surge en los ánimos más nacionalistas, en el sentido más puro posible, la objeción de que se pueda tratar, en definitiva, de un pérdida de identidad nacional a favor de una incierta realidad plurivocal y pluralista que culmina en una sintesis utópica. Claro es que la práctica comparada no admite nacionalismos por definición y de hecho, nos parece que la comparación es un acto de apertura frente al mundo, más que un acto de conocimiento de si. $\mathrm{O}$, si se quiere, ambas acciones son especulares y al conocer el mundo, se adquiere una más completa percepción de nuestra morada.

\section{Taxonomias y prácticas de la literatura comparada}

El método comparativo clásico propone problemáticamente unas taxonomias que son múltiples y varian según el aporte teórico-crítico de los estudiosos. Es obvio que las últimas décadas han registrado una substancial

\footnotetext{
"Véase Inirodwction to Latin America as Its Literature, Selected Papers of the XIVth Congress of the International Comparative Literafure Association, ed. by Maria Elena de Valdés, Mario Valdés and Richard A Young, Council on National Literatures Wodd Report editors, 1995.
} 


\section{AMÉRICA LATINA Y LA PRÁCTICA DE LA LITERATURA COMPARADA}

revolución en la literatura comparada, y esta asignatura, que es una de las formas «prácticas» de la teoría literaria y de la historia de la crítica, se ha corvertido en un recipiente, a veces malogrado, de relaciones entre literatura y medicina, literatura y biologia, literatura y culinaria. Sin embargo, es suficiente considerar las lineas de investigación clásica para mirar con otros opos el fenómeno literario latinoamericano en su emergencia universal.

Paul Van Tieghem (en 1931), Renato Poggioli (en un articulo de 1943) y Ulrich Weisstein (en la famosa Introducción a la Litenatara Comparada de 1968) concuerdan en reconocet que son cinco las categorias de estudios del comparativismo: 1) influencia e imitación; 2) recepción y efecto; 3) géneros; 4) historia de los temas y motivos; 5) época, periodo, generación y movimiento históricos. En los años setenta, Owen Aldridge y François Jost proponen otras configuraciones; después, René Wellek subraya la relación indispensable entre arte (pintura, escultura, etc.) y literatura; luego en los noventa, Cesare Segre, reconociendo una crisis del estudio de la literatura, declara la absoluta y fértil interrelación entre la teoria literaria $y$ la literatura comparada. La literatura comparada es finalmente teoría de la literatura.

El discurso se transforma en un escenario vasto, como de un teatro de marionetas, de las cuales no es dificil perder los hilos.

Asi, dentro del complejo universo latinoamericano, no seria anacrónico empezar el trabajo de investigación con las orientaciones primordiales y aplicar esta metodologia (hemos dicho que el comparativismo es, antes de todo un método de estudio e investigación) a la literntura de América Latina.

Consideremos, por ejemplo, como hipótesis de trabajo investigativo y como aplicación de dicha metodologia comparada, la cuestión de los géneros Literatios, que es una de las más antiguas y tambiên violentamente debatidas. A través de los géneros literarios se proponen unos modelos que cambian casi incesantemente $y$ que se sitúan en el polissistema de la literatura, con una significativa connotación que es la relación entre el éxto de un género y su público, la sociedad en la cual el género se ha desarrollado más.

Para América Latina, el campo de estudio sobre géneros literarios es excepcionalmente fecundo: la épica de Ollantay podría tener fuertes o inconscientes relaciones con la épica de Homero o, en general, con la forma épica que cada cultura mantiene como patrimonio celoso de su propio pasado, ya sea la 


\section{Bíagio D'Angelo}

griega, más difundida, según el preconcepto keurocéntricos o sea el épos de Gilgamesh o las épicas orientales con sus rasgos peculiares del honor y de la dignidad y de la unicidad del individuo.

La novela histórica, ¿cómo se ha desarrollado en Latinoamérica?, ¿de dónde deriva?, ¿cuáles son las formas más imponentes que han sido leidas por los autores latinoamericanos? Se podría encontrar un punto en común entre los textos de matriz histórica, es decir, textos donde ese funda la nación" (Forndational texts) como dice Doris Sommer, considerando a Gertrudis de Avellaneda, Bartoloné Mitré y Nataniel Aguirre.

Si la crónica se ha vuelto patrimonio nacional inmortal por obra del Inca Garcilaso de la Vega, en el Perú, çotros paises, otras culturas han aprendido una conciencia nacional y de pertenencia a un pueblo, a una historia, a una fe a través de la forma de una narración en Guatemala o en Colombia?

¿cLos géneros orientales más característicos encuentran una resonancin en un universo tan antagónico (aparentemente) como el mundo latinoamericano? ¿Hay, por ejemplo, viajes alegóricos como la Divina Commedia o como el Viaje a Occidente de Wu Ch'eng-en (siglo XVI)?

En la poesia quechua, recuerda Jesús Lara, existe una forma de poesía dialogada llamada mawaki que se cantaba en ocasión de fiestas en honor de la luna o para celebrar la buena cosecha y que era una forma korganizadà de poesia: un grupo iniciaba un canto y otro grupo, de frente, en círculo, del sexo opuesto, contestaba con otras palabras, más potentes, en forma de juego, de alegria o de polémica dionisiaca ${ }^{i}$. Es impresionante la cercanía de otras formas poéticas, similares al género aqui descrito, como el sema de los poetas escandinavos de la Edad Media (senna significaba 'batalla de palabras con estilo'), cuya única diferencia era que quienes combatian eman pastores-poetas, es decir, pastores con « conciencia de ser poetas\%. El resultado es extraordinario: aunque las diferencias resulten vistosas, los grupos que wse pelean» con palabras realizan un «discurso poéticon y subrayan el valor adecididamente poderoso, hasta mortals de las palabras. Es posible, entonces, admitir que la poesia coral ha sido la primera expresión cindividuabs antes que la forma lírica más especificamente personal, intima que conocemos.

"Vèuse I Lara, Loppoesia qwechwa, Fundo de Cuiturn Éconömiç, Méxuco, 1979. 


\section{AMÊRICA LATINA Y LA PRÁCTICÁ DE LA LITERATURA COMPARADA}

La novela histórica latinoamericana presenta fuertisimas connotaciones con el discurso de la mujer en la sociedad, mujer hetoína, mujer esclava, mujer libertadora y libertada: Amalia, de José Mármol, Manuela (novela de costumbres colombianas) de Eugenio Díaz Castro, o Luisia Miranda, de Eduarda Mansilla de Garcia: leyendo estas novelas, cuyos resultados estilisticos son discutibles, no estamos lejos de un guión cinematográfico, que sin duda habría gustado al guionista boliviano de radionovelas de La tía Jutia y ol escribidor de Mario Vargas Llosa.

No se pueden enumerar las mujeres que se han realizado poéticamente a través de la forma de distracción más burguesa de la época romántica, la novela, y son tan numerosas que en América Latina merecen un estudio crítico cuidado, que tenga cuenta de los efectos de la novela epistolar de Richardson o la novela gótica de Ann Radcliffe y Mary Shelley. Asi, para leer a Juana Manuela Gorriti, Mercedes Cabello de Carbonera, Clorinda Matto de Turner, Virgina Auber Noya se podria realizar un estudio del universo social de Centro y Sur América en el siglo XIX, que sorprenderia pot su visión moderna ante litteram no sóla sobre la mujer sino también sobre la evolución del hecho literario en sí.

\section{La práctica de la literatura comparada en América Latina}

Esbozar la historia de la literatura comparada en Latinoamérica es como delinear los contornos de una tentativa en lucha constante a favor de una apertura a la literatura universal, no obstante exista el riesgo de malinterpretar tal apertura con una robusta, tal vez inconsciente, recuperación intelectual nacionalista. Scrá indispensable, entonces, limitarse a tres de las experiencias básicas de la metodologia comparada: nos estamos refiriendo al mundo académico de Brasil, de Uruguay y de Argentina, sobre todo. Como se podri temarcar, dibujar un mapa de la adhesión a la literatura comparada coincide con el ejercicio crítico y con la profunda intuición de algunos teóricos de la literatura. Es decir, la literatura comparada nace como labor dialéctica entre las definiciones de una teoria y la necesidad obligatoria de la práctica literatia, para que comparar no se transforme en una actividad fríamente algoritrnica. De hecho, muchos de los grandes nombres de la crítica sudamericana han sido comparatistas (algunos de ellos, comparatstas en nuce) o ban optado por un horizonte intelectual abierto a la 


\section{Biagio D'Angela}

universalidad. El caso rioplatense es el más evidente $y$ más próspero de intuiciones, fundamentos teóricos, valiosos estudios de la materia. Sin embargo, una serie de coincidencias fortuitas y debidas a factores no meramente liretarios, coma aquellos económico-sociales, ha producido un vastisimo interés hacia la literatura comparada de forma especial en el continente brasileño, tal vez con centros de investigaciones distantes de Buenos Aires y Montevideo. Puede resultar importante subrayar que a menudo la práctica de la literatura comparada ha sido reforzada por una base economica favorecedora, elemento, éste, que ha culminado en una acusación de práctica aristocrática. La influencia de la así llamada kestacións del comparativismo francés, cuyo jefe era el recién fallecido René Etiemble, habia podido permitir esta conclusión. Sin embargo, las iluminadas acciones concertadas de ministerios y universidades en ciertos paises del mundo europeo y oriental, tal vez menos desartollados o menos zcéntricos", contrastan con este jucia y se oponen a una reducción regionalista de la literatura nacional (piénsese en países como India, Hungría, Estonia, Corea, Grecia y muchos más, que poseen actualmente algunas de las más activas asociaciones nacionales de literatura comparada).

La actitud tradicional de la critica comparada esporádica y asistemática, que habia considerndo las primeras historias literarias del continente latinoamericano, tendrâ su institucionalización definitiva en Brasil con una licenciatura (Groduagäo) y con una maestria (Pos-graduafão) en la universidad de São Paulo, por obra de António Cándido en 1962. Y fue siempre este último quien declaróla madurez académica de los estudios comparados en Brasil en ocasión de la apertura del I congreso de la Asociación Brasilera de Literatura Comparada (ABRALIC), única caso de asociación extraordinariamente activa en el hemisferio sur; " $\mathrm{Ya}_{2}$ bace cuarenta años habia dicho que estudiar la literatura brasilera es estudiar la literatura comparadan. Con esta frase provocativa, Cándido inauguraba una serie de estudios de influencia europea en Brasil, que siguen aún ahora en algunos centros de investigaciones, primero el dirigido por las profesoras Sandra Nitrini y Leyla Perrone-Moisés, de la Universidade de São Paulo. Entre las obras criticas más discutidas en el marco de la práctica comparada, se deben recotdar los ensayos de Silviano Santiago de 1970, Eqa, autor de Mudane Bovary, O entro-lugar do disarso latino-americano ('El entre-lugar del discurso latinoamericano') de 1971 ; $A$ pesar de dependente, smiversal ('Aunque 


\section{AMÉRICA LATINA Y LA PRÁCTICA DE LA LITERATURA COMPARADA}

dependiente, universal') de 1980. Mientras que en este último, Santiago desarrolla la idea de la ruptura conceptual de la importancia absoluta de la literatura hegemónica a favor de la (muevas literatura a estudiar, en el texto de 1971 concibe el término de sentre-lugaps para afirmar que estudiar la literatura de Brasil es no una postura aislacionista, sino un descubrimien to de la propia cultura a partir de objetos teóricos y obras de ficción de autores de distintas nacionalidades. Será una de las bases de la teoría comparada brasilera, seguida por Luis Costa Lima, que profundiza en términos socioculturales las formas del imaginario recortado entre el pensamiento occidental y el espacio nacional, en una especie de xinterlocucións con la cultura extranjera.

En ocasión del I Seminario Latinoamericano de Literatuma Comparada, realizado en Porto Alegre, fue creada en 1986 ABRAIIC, la asociación nacional de literatura comparada, bajo la responsabilidad de Tania Franco Carvalhal, ferviente curadora de innombrables eventos relacionados con la difusión de la literatura comparada. ABRALIC se reúne, desde 1986, cada dos años, con un éxito siempre creciente de público. La asociación se ha revelado en el tiempo como una posibilidad efectiva de reflexión latinoamericana a propósito de las condiciones de producción y recepción de la literatura en el continente. Entre los temas tratados en estos encuentros destacan ointertextualidad e interdisciplinatiedadn, 《Literatura y memoria culturals, "Cánones y contextos", "Lireratura y mediaciones mediáticas»; este último se ha realizado este año en Belo Horizonte, en la Universidad Federal de Minais Gerais. ABRALIC publica, además, las Actas de los Congresos y una Revista brasilera de literatura iomparada, bajo la dirección de Eneida Maria de Souza. Tania Carvalhal, en cambio, posec el mérito de haber difundido y reintroducido la discusión sobre ciertas cuestiones teóricas y metodológicas de la literatura comparada, reafirmando la importancia en nuestra época y el progreso efectuado por esa disciplina con dos ensayos muy interesantes: Literatura comparada, de 1986, y Literatura comparada. Textos fundadores, de 1994, escrito con la colaboración de Eduardo Coutinho, de la Universidad Federal de Rio de Janeiro. En esos libros, a nuestro parecer, capitales, Tania Carvalhal explica que el elemento nacional o macro-regional, como instancia de identidad literaria, adquiere su propio valor de referencialidad cuando está atravesado por la heterogeneidad que lo constituye y que lo contrasta por su originalidad en el conjunto de obras en que se instala. 


\section{Biagio D'Angelo}

Afurma Lisa Block de Behar que da actualidad uruguaya ha impuesto en los útimos tiempos una obsesionante definición de la identidad nacionals. Esta autecelebración, que se opone a la práctica de la «comparación» literaria, ha contribuido a dispersar la ya discontinua doctrina literario-filosófica según la cual se habian dado los fundamentos teóticos de la literatura comparada. Una de las más felices expresiones sobre esta metodologia viene justamente de uno de los intelectuales más finos y sensibles de este pais, Carlos Real de Azúa, que define el continente latinoamericano como un ktrasatlänticon, o mejor, de aorigen trasatlánticon. Detrás de esta metáfora se trataria, más allá de los confines geográficos, de un origen que alude a regiones, mundos, culturas del otro lado del océano, un klargo barco destinado a atravesar todo mats: también el puerto de Montevideo se trans forma en una metáfora de lanzamiento hacia un mundo que se desea re-descubrir, anhelado, misteriosamente reconocido. Este trasatlántico buscará un origen espiritual e idealista en una Europa que Real de Azua llama «a gtan presenciau. Su imperio - declata Real de Azúa- es absoluto en lo económico, en lo cultural y en lo humano. Europeas son las ideas; muestra economía depende de las alternativas de sus ciclos y de la intensidad de sus compras..... Un verdadero bofetón moral a todos aquellos intelectuales que rechazan la pertenencia o la derivación eurocéntrica. Un cierto comparatismo avant la lettre se encuentra en uno de los textos fundadores de la literatura y crítica uruguayas, y de la conciencia ideológica latinoamericana, Ariel de José Enrique Rodó, de 1900, en que se postula ya la necesidad de escribir un texto de teoría líteraria para brindar los fundamentos de la universalidad en la práctica del conocimiento literario. Una excepcional apertura a los clásicos europeos y el reconocerlos cuna del pensamiento universal caracteriza el escrito de Rodó, según una sheterogénea arqueologia de conocimiento transtextuals.

Con diferentes iniciativas como la publicación de las revistas Marcba, Nimera, "Mundo nuevo" (aunque fuera de Uruguay) y de algunos números de la Revista Iberamericana (en Estados Unidos), es Emir Rodriguez Monegal el ecuménico profeta de la práctica comparativa en Montevideo (y no sólo alli) con cursos de estética literaria, teoría de la literatura, metodología de la literatura comparada. El critico presenta sobte todo un inventario de literatura universalen que se resaltan autores como Proust, Henry James, Joyce, Kaflsa, Gide, el latimoamerizana Faulkner, Borges, Neruda, Quiroga y Onetti A Rodriguez 


\section{AMÉRICA LATINA Y LA PRÁCTICA DE LA LITERATURA COMPARADA}

Monegal se opondrâ la visión social comprometida de Ángel Rama en los mismos años de tmabjo.

Finalmente, en 1985, para celebrar la caida de la dictadura militar, Lisa Block de Behar inaugura una cátedra de teoria literaria en el departamento de literatura de la Universidad de la República de Montevideo con una serie de importantísimos seminarios y conferencias con huéspedes de honor de gran fama: para empezar, Gérard Genette, que presentó algunas conferencias de su trabajo inédito sobre el paratexto; luego, Rodríguez Monegal, Haroldo de Campos, J. Hillis Miller, Geoffrey Hartman y sobre todo Jacques Derrida, en honor del cual se publicó una colección de sus ensayos con el titulo indicativo de Diseminario: la deconstración, otro descubrimiento de América de 1985. En el mismo tiempo fue fundada la cátedra de Semiótica, llamada ahora Semiótica y Teoria de la Interpretación y dirigida siempre por Lisa Block de Behar. En 1988, gracias al apoyo de ABRALIC, se realiza la formación de la Asociación Uruguaya de Literatura Comparada (AULICO) que publica con entusiasma un volumen intitula do Términos de comparación. Los estudios literarios entre historiary teorias y prepara en 1989 la fundación del Centro Cultural Internacional de Salto, una ciudad uruguaya equidistante de Brasil y Argentina, y donde se realizó un congreso internacional sobre Borges en ocasión del decimoquinto aniversario de la publicación del famoso cuento borgiano «Tlön, Uqbar, Orbis Tertius», compuesto - como dice la didascalia final - justamente en esta ciudad, Salto. A parte de otros coloquios, uno sobre Bioy Casares en 1991 y otro sobre Lautréamont y Laforgue en 1992, AULICO no opera actualmente, y Lisa Block habla de una siempre mayor ofuscación intelectual que ha amenazado definitivamente el mundo académico porteño.

Es el mismo caso de Argentina, sin contar con los problemas que están afligiendo al pais en estos últimos meses. El primer centro de estudios comparatistas fue el Centro de Literatura Comparada de la Universidad de Cuyo, en 1976, dirigido por Nicolás Dornheim, que con muchos esfuerzos publica, gracias al apoyo de numerosos investigadores internacionales, los Boletines de Literatura Comparada. En la huella del centro de Cuyo, fue creado otro centro en la Universidad Católica de Buenos Aires, por interés de la profesora Maria Teresa Maiorana, una especialista de literatura francesa que ha concentrado sus intereses sobre la influencia europea en la producción de Rubén Darío. Finalmente, se 


\section{Bíagio D'Angelo}

encuentra la creación de la Asociación Argentina de Literatura Comparada que, después de un periodo dificil, está retomando su labor con un coloquio anual, gracias al interés académico y al aporte internacional de Tania Franco Carvalhal de la Universidad Rio Grande do Sul.

El argumento se dilata de forma tentacular. Nos quedan algunas alusiones a la situación del Perú, pais que no ha tenido una particular predisposición por la práctica de la literatura comparada. Es dificil, y mucho más complejo para un extranjero, explicar o detallar las razones de este silencio que se mantiene, pues to que el único curso oficial de Literatura Comparada se dicta en la Pontificia Universidad Católica y es un curso electivo. Claro que sería un buen auspicio una renovación del curriculo académico con la obligatoriedad de esta asignatura, pero sobre todo la presencia de maestros y docentes que, bajo un empuje teórico destacable, puedan transformat la conciencia atústica nacional en un trasatlántico (retomo la imagen poética de Real de Azúa) abierto a los estudios y a la profundización de temáticas teórico-prácticas ya recorridas, pero indudablemente ricas todavia de motivos de estudios. Sin olvidar los importantes. aportes críticos actuales de teóricos de la Universidad Nacional Mayor de San Marcos como Carlos García Bedoja y Miguel Ángel Huamán, que han dedicado sus esfuerzos a la reflexión del hecho literario como epifenómeno de la cultura de una nación y han insistido en la lucha por la afirmación de la base teórica del fenómeno mismo de la literatura, dos son los autores que aquí se quiere mencionar: Estuardo Nuñez y Antonio Cornejo Polar Como catedrático de San Marcos, Estuardo Nuñez dirigió sus investigaciones hacia la literatura comparada y como resultado de ello produjo una docena de volúmenes consagrados a revelar, traducir y editar a los autores y viajeros que han escrito sobre las realidades peruanas e hispanoamericanas entre los siglos XVI y XX. Un compendio del panorama de estos viajeros integra su magnifico volumen Viajes y viajeras extranjeros sobre el Perzi. Nuñez ha tratado también de fijar los contactos de la Litetatura peruana con las letras de Alemania, Gran Bretaria, Estados Unidos, Italia y Francia, así como estudiar la contribución a la literatura peruana de escritores, poetas y novelistas que viajaron por diversos paises del mundo y que optaron de esta forma pot el proceso transcultural de la práctica comparada a través de su métoda más celebrado, es decir, el de los estudios de las fuentes histórico-literarias y de sus influencias: 


\section{AMÉRICA LATINA Y LA PRÁCTICA DE LA LITERATURA COMPARADA}

A partir de su estudio sobre da formación de la tradición literarta peruanay, de 1989, Antonio Cornejo Polar ha subrayado la presencia inquietante y olvidada de «abismos de inestabilidad y polimorfismon producida por una mezcla compleja de clases sociales y grupos étnicos en el Perú, que será explicitada en un concepto caro a la opción comparativa que se ocupa de literatura (o literaturas) latinoamericana(s): el de la heterogeneidad. Polemizando con la aplicación eurocéntrica que enfatiza la falsa unidad conceptual de homogeneidad cultural en el continente latinoamericano, equivoco debido quizás a una aplicación errada de una noción aparentemente unitaria del ideal romántico europeo, Cornejo Polar crítica la restricción de la idea de que la literatura latinoamericana corresponda casi exclusivamente a la producción escrita en lenguas europeas, seguin el famoso canon accidental de normas estéticas que marginaliza forzosamente la producción de la tradición autóctona y popular, Cornejo Polar refuta la concepción superficialmente monolítica de una presumida kúnica» literatura latinoamericana por causa de una ridicula simplificación de la compleja realidad del continente mismo. Escribe Cornejo Polar en Escribir en el aire de 1994:

[El concepto de literaturas heterogéneas] da razón de los procesos de producción de literaturas en las que se intersectan conflictivamente dos o más universos socioculturales [...], poniendo énfasis en la diversa y encontrada filiación de las instancias más importantes de tales procesos (emisor/discurso/texto/referente/receptor, por ejemplo) [....] En todos los casos me interesa la indole excepcionalmente compleja de una literatura (entendida en su sentido más amplio) que funciona en los bordes de sistemas disonantes, a veces incompatibles entre si $[\ldots]^{11}$

No cabe duda de que el nuevo futuro comparatista peruano debe nacer de la sintesis de las dos posturas críticas ahora mencionadas que justifican asi el camino práctico y el fundamento teórico que la literatura comparada necesita para desarrollarse como descubrimiento de novedades y apertura y presencia en el universo cultural del mundo, sobrepasando las paradojas de un discurso nacionalista que cierra las puertas y los ojos al devenir humano.

\footnotetext{
"A. Comejo Polar, Escribir en el aire. Ensgyos solure la beterogeneidad socio-cultural en las filendiwras anditias, Lirra, Honzonte, 1994, pp. 16-17.
} 


\section{Biagio D'Angelo}

\section{Conclusión}

Ejercer literatura comparada es una auténtica vocación, más allá de las normales preocupaciones teóticas y metodológicas, sin las cuales una actitud de observación de lo real degradaria en instinto personalista. Anna Balakian to recordaba en un articulo al intentar explicar, retrocediendo en la memoria, los motivos que le habían llevado a convertirse en comparatista ${ }^{12}$. Se podría clasificar al comparatista como una mezcla entre un aventurero $y$ un misionero, porque trata ambiciosamente, $y$ quizás también inconscientemente, de contribuir a través de sus cartas a encontrar (o reencontrar) una forma moderna de humanismo. $Y$ no se trata sólo de estos males éticos, auténticas plagas sociales como la intolerancia, el racismo, la exclusión social, el nacionalismo, que todos conocen. El comparatista es, fundamentalmente, un idealista que cree en la presencia de factores espirituales y existenciales comunes a todos los hombres, pero que se expresan en la literatura, como cualquier arte y en la existencia, con modalidades diferentes y originales. A la pregunta ¿quién es entonces el comparatista?, Julien Gracq afirmaba con su sarcasmo habitual que dos comparatistas son "perforadores" (perceurs) de fronteras, que construyen puentes entre orillas que secularmente se ignoran, aunque tal vez se trata más bien de la perspectiva que de la circulacións.

Finalmente, podremos definirlo, de forma irónica, como un incurable romántico, que desafia al mundo porque es consciente de la necesidad de su don de observador critico y constructivo, pero que también sabe adaptarse a los tiernpos y a la historia, sin plegarse nunca a los poderes dominantes. Eri la incertidumbre dictada por la época de la postmodernidad, éste aún puede decit que se debe obligatoriamente afrontar la contemporaneidad con un doble criterio sumergir el conocimsento en la propia cultura indigena y referirse con espíritu curioso y abicrto al mundo múltiple que lo alberga.

\footnotetext{
"A. Balakian, "How and Why I Became a Comparatist", en Bailding a Profession: Aurobiographical Perspections ont the Begennings of Comparative Litenature in the United Stutes, ed. Luonel Gussman and Mihai 1. Spacosi, Albany, Stare Unversity of New York Prese, 1994, pp. 75.87.
} 


\section{AMẼRICA LATINA Y LA PRÁCTICA DE LA LITERATURA COMPARADA}

\section{REFERENCIAS BIBLIOGRÁFICAS}

1994, Balakian, Anna, "How and Why I Became a Comparatist" en Building a Profession: Autobiograpbical Perspectives on the Beginnings of Comparative Litorature in the United States, ed. Lionel Gossman and Mihai I. Spariosu, Albany, State University of New York Press, pp. 75-87.

1999, Birus, Heinrich, "Main Features of Goethe's Conception of World Literature" en Comparative Literature Now: Theories and Practice/ La litterature comparada à l'beure actuelle. Théories y pratigues, ed. S. Tötösy de Zepetnek, Milan V. Dimic and Irene Sywenky, Paris, Honoré Champion, pp. 31-40,

1964, Cioranescu, Alexandre, Prinapios de literatura comparada, La Universidad de Laguna.

1994 Cornejo Polar, Antonio, Escribir sn el airc. Ensayos sobre ka heterogeneidad sociocultural en las literaturas andinas, Lima, Horizonte.

1985 Guillén, Claudio, Entre lo zuo y lo diverso. Introducción a la literatura comparada, Batcelona, Editorial Crítica.

2002 Hopkins, Eduardo ed., "La literatura comparada: un desafio y una necesidad", Homenaje a Luis Jaime Cistems, vol. II, Lima: Fondo Editorial de la PUCP,pp. 813-825.

1979 Lara, Jesús La poestá quectua, Fondo de Cultura Económica, Méxica

1982 Marino, Adrian Etiemble out le comparatisme militant, Paris, Gallimard.

1983 Paz, Octavio Pintado en Mexico, El País, Madrid, 7 de noviembre.

1995 De Valdés Maria Elena, Young Mario, Richard A. eds, Latin. America as It Literature, Selected Papers of the XIVth Congress of the International Comparative Litera-ture Association, Council on National Literatures World Report editors. 